\title{
Asymmetric Price-Benefits Auctions
}

\author{
M. Angeles de Frutos ${ }^{1}$ \\ Department of Economics, Universidad Carlos III de Madrid, C. Madrid, 126. \\ 28903 Getafe, Madrid, Spain
}

This paper studies the performance of two auction procedures for allocating the assets of a dissolving partnership when partners' valuations for the assets are independent and asymmetrically distributed, with one partner reputed to be more interested in the assets to be divided. We provide results on existence, and uniqueness of the equilibrium induced by these auctions. Comparative statics are developed, especially the differences between relative efficiency and revenue from the two auctions. Journal of Economic Literature Classification Numbers: C72, D39, D44, D52, D74, D82.

\section{INTRODUCTION}

In many auctions bidders split the revenue generated by the auction. This is the case, for instance, whenever auctions are used to dissolve a partnership (a joint venture or a marriage) or when they are used to settle the competition between raider firms seeking to take over a target firm. We consider two-party partnership in which each partner is endowed with an equal share of the assets. If the partnership dissolves, assets are divided by using $k+1$-price auctions. ${ }^{2}$ In these auctions, the players submit sealed bids, and the good is transferred to the highest bidder who pays each of the others a price that is an exogenously given convex combination of the highest bid and the second highest bid.

${ }^{1}$ This paper was partially written during my stay at Boston University. I am indebted to the economics department at B.U. for the support received during this work. I have benefited from comments by Robert Rosenthal, Svika Neeman, Deborah Minehart, and Cesar Martinelli. Comments by an anonymous referee have substantially improved the paper. Financial support by a Fulbright grant from the Ministerio de Educacion of Spain and by grant DGICYT PB96-0118 is gratefully acknowledged. Any error is my exclusive responsibility. E-mail: frutos@eco.uc3m.es

${ }^{2}$ These auctions were first introduced in Cramton et al. (1987; CGK, for short) and are also studied, for the common-values case, by Engelbrecht-Wiggans (1994). 
This paper focusses on two of the above auctions: the winner's bid auction (WBA), in which the winning bidder pays half of his bid to the losing bidder, and the loser's bid auction (LBA), in which the winning bidder pays half of the losing bidder's bid to the losing bidder. ${ }^{3}$ In contrast with previous work on these auctions, we assume that the agents' valuations are independently and asymmetrically distributed on some common real interval. Further, we assume that one partner is reputed to be more interested in the assets; in the Maskin and Riley (1999) terms, there will be a weak and a strong bidder. As in standard auctions, asymmetries among bidders are quite common. Asymmetries may be due to different budget constraints, or to institutional arrangement. In divorce settlements, for example, courts give more to the financially weaker party. In a takeover, asymmetries may arise due to different synergies or managerial skills.

In both auction formats, bids are closer to valuations than in standard first and second price auctions. Note that bidders have countervailing incentives; bidders are tempted to exaggerate their true valuations if they were to sell, whereas they will want to understate if they were to purchase. Under asymmetric distributions, the bidding equilibrium functions of these auctions consist of continuously differentiable and strictly monotonically increasing strategies which are characterized as solutions of a system of differential equations with two boundary conditions. Through a change of variables, we show that the equations and boundary conditions that characterize the equilibrium of these auctions are identical to the equations and boundary conditions that characterize the equilibrium of certain asymmetric first price auctions. This fact allows us to apply the results about existence and uniqueness developed by Lebrun (1999; 1998) and Maskin and Riley (1999) for standard first price auctions with heterogeneous bidders. By applying their results, we show that the partner that is reputed to be more interested in the asset will bid more conservatively, irrespective of the auction in use. Nevertheless, she is more likely to win the auction. In contrast to the symmetric case, in both of these auctions the resulting allocation need not be efficient.

Under the WBA, we show that the bidders always shade their bids whereas under the LBA they always submit bids in excess of their valuation. In the latter case, overbidding may result in the winning party losing money. In the context of a takeover, this result is consistent with the observation that the stock price of a successful raider sometimes falls dramatically. ${ }^{4}$ Two numerical examples show that the LBA generates larger

\footnotetext{
${ }^{3}$ The WBA and LBA have been studied for the symmetric case by McAfee (1992). We have adopted here his terminology. In Bulow et al. (1999), for the takeover application, the authors refer to these auctions as first and second price auctions, respectively.

${ }^{4}$ This fact has been pointed out by Bernhardt (1995) for the LBA equilibrium bids of two raider firms with symmetric valuations but with different initial stakes in the target firm.
} 
expected selling price than the WBA. In one of these examples the LBA is more inefficient than the WBA. Both of these results are consistent with results in Bulow et al. (1999) who study takeovers when the takeover target has a common value to the bidders. They show that if the bidders have equal initial stakes, or toeholds, in the target firm, and if the signal distributions are symmetric, then the expected sale price is higher in a LBA. Nevertheless, the probability that the bidder with the higher signal wins the auction is greater in a WBA. ${ }^{5}$

We compare the revenue from the two auctions. For the symmetric case, we show that the LBA generates larger expected selling price than the WBA. In the asymmetric case, we provide sufficient conditions for this result to hold. Our results are consistent with Maskin and Riley (1985) claim that second-price auctions are more likely to generate more revenue than first-price auctions when, across bidders, distributions have different shapes but approximately the same support.

The paper is organized as follows. Section 2 presents the model and assumptions. Section 3 contains the results on existence, uniqueness and properties of the equilibrium induced by the WBA and the LBA. Section 4 provides a revenue comparison among them. Section 5 concludes. Finally, some of the proofs are included in the Appendix.

\section{THE MODEL}

Two risk-neutral partners $(i=1,2)$ want to dissolve their partnership. It is assumed that each partner has an equal share of the asset to be traded. In the case of takeovers, two risk-neutral bidders with equal toeholds compete to acquire a company. Partner $i$ has a valuation for the entire object of $v_{i}$ which is only known to him. Valuations for the asset are independent but asymmetrically distributed. Partner 1's beliefs about the value that partner 2 places in the good are drawn from the c.d.f. $F_{2}(v)$, and partner 2's beliefs about the valuations of partner 1 are summarized by the c.d.f. $F_{1}(v)$. These distributions are common knowledge.

In order to compare the properties of the two auctions that are studied in this paper, we consider the following assumptions related to the players' beliefs:

(A1) Both cumulative distribution functions are continuous and differentiable over their common support $[0, \bar{v}]$. Their density functions $f_{1}, f_{2}$ are locally bounded away from zero over $(0, \bar{v}]$.

${ }^{5}$ For this last result to hold the assumption of equal toeholds is not required. 
(A2) Partner 2's valuations are higher in the sense of first order stochastic dominance, that is, $F_{2}(v)<F_{1}(v)$ for all $v \in(0, \bar{v})$.

(A3) Partner 2's valuations are higher in the sense of reverse hazard rates dominance, i.e. $F_{2}(v) / F_{1}(v)$ is strictly increasing in $v$ for all $v \in(0, \bar{v})$.

(A4) Partner 2's valuations are higher in the sense of hazard rates dominance, that is, $H_{1}(v)=f_{1}(v) /\left[1-F_{1}(v)\right]>H_{2}(v)=f_{2}(v) /\left[1-F_{2}(v)\right]$ for all $v \in(0, \bar{v})$.

(A2) is an assumption of stochastic dominance of $F_{1}$. Under this assumption, the probability distribution $F_{1}$ gives less weight to high values of $v$ than $F_{2}$ does. This implies that bidder 2 is reputed to be more likely interested in the asset than bidder 1. (A3) is an assumption of reverse hazard rate dominance of bidder 2's valuations, which is a stronger version of stochastic dominance. This assumption has been used by Maskin and Riley (1999) to ensure that in a first price auction the more optimistic buyer is the less "aggressive bidder" (we are using their terminology here). Finally, (A4) is an assumption of hazard rates dominance which is also a stronger version of stochastic dominance. It states that bidder 1 has a higher probability of having a low valuation conditional on valuations being above $v$. A sufficient condition for (A3) and (A4) simultaneously hold, and therefore (A2), is that bidder 2's valuations be higher in the sense of monotone likelihood ratio dominance (for an argument see Shaked and Shanthikumar, 1994).

\section{3. $k+1$ PRICE AUCTIONS}

In a $k+1$ price auction each player submits a sealed bid. The bidder with the highest bid, $b_{w}$, gets the object and has to pay the other $0.5\left(k b_{l}+\right.$ $\left.(1-k) b_{w}\right)$, where $b_{l}$ denotes the loser's bid. In particular, we will study the following two members of this class of auctions: the winner's bid auction (WBA) that corresponds to the case $k=0$ and the loser's bid auction (LBA) where $k=1$.

Suppose that the object has a true value $v_{i}$ for bidder $i$ and that bids $b_{i}$ and $b_{j}$ are submitted by bidders $i$ and $j$, respectively, $i, j=1,2, i \neq j$. Then bidder $i$ 's payoff is

$$
u_{i}\left(v_{i}, b_{i}, b_{j}\right)= \begin{cases}v_{i}-0.5\left(k b_{j}+(1-k) b_{i}\right), & \text { if } b_{i}>b_{j} \\ 0.5\left(k b_{i}+(1-k) b_{j}\right), & \text { if } b_{i}<b_{j} \\ 0.5 v_{i} & \text { if } b_{i}=b_{j}\end{cases}
$$




\subsection{The Winner's Bid Auction}

The bidder with the highest bid wins the auction and pays half of her bid to the other bidder. Ties are resolved by the flip of a coin.

We will show that there exists a unique equilibrium for this auction and that this equilibrium is such that the bidder that is reputed more interested in the asset bids less aggressively.

LEMMA 1. Bidders' equilibrium strategies must be pure strategies $B_{1}\left(v_{1}\right)$ and $B_{2}\left(v_{2}\right)$ that are continuous and strictly increasing functions of their types with $B_{1}(0)=B_{2}(0)=0$ and $B_{1}(\bar{v})=B_{2}(\bar{v})<\bar{v}$.

Proof. See appendix.

Rather than attempting to solve directly for the equilibrium bid functions, it is convenient to define a pair of inverse bid functions $v_{i}=w_{i}(b) \equiv$ $B_{i}^{-1}(b)$, for $i=1,2$.

Since strategies are increasing, if bidder $i$ bids $b$ and bidder $j$ follows the strategy $B_{j}$ then her expected payoff is

$$
U_{i}\left(v_{i}, b, B_{j}\right)=\left(v_{i}-\frac{b}{2}\right) F_{j}\left(B_{j}^{-1}(b)\right)+\int_{B_{j}^{-1}(b)}^{\bar{v}} 0.5 B_{j}\left(v_{j}\right) d F_{j}\left(v_{j}\right)
$$

At an interior solution ${ }^{6}$ partner $i$ 's bid solves the first-order condition

$$
\left(v_{i}-b\right) \frac{\partial B_{j}^{-1}(b)}{\partial b} f_{j}\left(B_{j}^{-1}(b)\right)=0.5 F_{j}\left(B_{j}^{-1}(b)\right) .
$$

The right-hand side is the marginal cost of bidding more when $i$ wins. The left-hand side represents the marginal revenue for increasing the bid and winning. Notice that this first order condition implies that the inverse bid functions $\left(w_{1}, w_{2}\right), w_{i}(b)=B_{i}^{-1}(b)$, must be solutions of the system of differential equations,

$$
2\left(w_{i}-b\right) f_{j}\left(w_{j}(b)\right) w_{j}^{\prime}(b)-F_{j}\left(w_{j}(b)\right)=0 .
$$

System (1) and the boundary conditions define equilibrium strategies if these conditions define a best decision for each bidder. We check this additional condition as part of the proof in the following theorem which characterizes the equilibrium of the WBA.

${ }^{6}$ Making this argument assumes $B_{j}^{-1}(b)$ is differentiable. Strictly it can be shown that the right and left derivative exist and coincide. The arguments are similar to those used in Bulow et al. (1999). 
THEOREM 1. A pair of strategies $\left(B_{1}, B_{2}\right)$ is an equilibrium for the WBA if and only if the strategies are pure, the bid functions are strictly increasing and differentiable, with $B_{i}(v)<v$ for all $v \in(0, \bar{v}]$, and there exists $\bar{b}, 0<\bar{b}<\bar{v}$, such that the inverse bid functions $\left(w_{1}, w_{2}\right), w_{i}(b)=B_{i}^{-1}(b)$, are solutions over the interval $[0, \bar{b}]$ of the system of differential equations,

$$
2\left(w_{i}-b\right) f_{j}\left(w_{j}(b)\right) w_{j}^{\prime}(b)-F_{j}\left(w_{j}(b)\right)=0
$$

satisfying the boundary condition $w_{i}(0)=0, w_{i}(\bar{b})=\bar{v}$ for $i=1,2$.

\section{Proof. See Appendix.}

We will now show that such an equilibrium exists and that under (A3) it is unique.

THEOREM 2. If (A1) holds then there exists a Bayesian equilibrium for the WBA. If, in addition, (A3) holds, then the equilibrium is unique.

Proof. Equations in system (1) can be rewritten as follows:

$$
\frac{d}{d b} \ln F_{j}\left(w_{j}(b)\right)^{2}=\frac{1}{w_{i}(b)-b}, \quad i=1,2, \quad i \neq j
$$

They are thus identical to the equations of the equilibrium of a first price auction with two bidders when their valuations are drawn from $G_{1}=$ $F_{1}^{2}$ and $G_{2}=F_{2}^{2}$. The boundary conditions $w_{i}(0)=0, w_{i}(\bar{b})=\bar{v}$, where $i=1,2$ and $\bar{b}$ is a parameter belonging to $(0, \bar{v})$, are also identical in both cases. The existence and uniqueness results of the equilibrium of the WBA then follow immediately from the known results for the first price auctions with heterogeneous bidders. In particular, existence follows from Theorem 2 in Lebrun (1999). A sufficient condition for uniqueness [see Corollary 4 in Lebrun (1999)] is reverse hazard rate dominance. Since $(d / d v)\left(F_{2}(v) / F_{1}(v)\right)>0$ by (A3) then $(d / d v)\left(G_{2}(v) / G_{1}(v)\right)>0$ holds, and uniqueness follows.

The following proposition shows some of the properties of the equilibrium induced by the winner bid auction.

Proposition 1. Under (A3) if $\left(B_{1}, B_{2}\right)$ is an equilibrium strategy profile to a WBA then

(i) $B_{1}(v)>B_{2}(v)$, for all $v \in(0, \bar{v})$,

(ii) The distribution of bidder 2's bids stochastically dominates the distribution of bidder 1's bids. 
Proof. The equations that determine the equilibrium for the WBA are identical to the equations of the equilibrium of a first price auction with two bidders when their valuations are drawn from $G_{1}=F_{1}^{2}$ and $G_{2}=F_{2}^{2}$. From Maskin and Riley (1999) (see Propositions 2.2. and 2.4) we know that $(d / d v)\left(G_{2}(v) / G_{1}(v)\right)>0$ implies $w_{1}(b)<w_{2}(b)$ and $F_{2}\left(w_{2}(b)\right)<F_{1}\left(w_{1}(b)\right)$ for all $b \in(0, \bar{b})$. Since (A3) guarantees that $(d / d v)\left(G_{2}(v) / G_{1}(v)\right)>0$ holds, the proposition follows.

In the WBA the reputed more interested bidder will bid "less aggressively". Since bidder 1 faces a fiercer competition, the one from bidder 2 that is reputed to be very interested in the asset to be sold, it is natural that she bids "more aggressively". She is in a worse position than bidder 2 because she faces a bid probability distribution that gives high weights to high values, consequently one would expect her payoffs to be smaller.

\subsection{The Loser's Bid Auction}

The bidder with the highest bid wins the auction and pays half the loser bid to the other bidder. Ties are resolved by the flip of a coin.

We will show that there exists a unique equilibrium for this auction. To prove this result one must first show that equilibrium bid strategies $\left(B_{1}\left(v_{1}\right), B_{2}\left(v_{2}\right)\right)$ are strictly increasing with differentiable inverse bid functions. If $B_{i}$ is strictly increasing, bidder $i$ 's expected payoffs when bidder $j$ follows strategy $B_{j}$ are

$$
U_{i}\left(v_{i}, b, B_{j}\right)=\int_{0}^{B_{j}^{-1}(b)}\left(v_{i}-\frac{B_{j}\left(v_{j}\right)}{2}\right) d F_{j}\left(v_{j}\right)+\frac{b}{2}\left(1-F_{j}\left(B_{j}^{-1}(b)\right)\right) .
$$

At an interior solution, bidder $i$ 's bid, $b=B_{i}\left(v_{i}\right)$, satisfies

$$
\left(b-v_{i}\right) f_{j}\left(B_{j}^{-1}(b)\right) l_{j}^{\prime}(b)=0.5\left(1-F_{j}\left(B_{j}^{-1}(b)\right)\right) .
$$

Thus, in equilibrium, the marginal cost from overbidding, $b-v_{i}$, has to be equal to the marginal increase in the payment that partner $i$ gets when she loses. This equation can be derived by differentiating $U_{i}\left(v_{i}, b, B_{j}\right)$ with respect to $b$ and setting that derivative to zero.

To obtain the appropriate boundary conditions assume first, w.l.o.g., $B_{1}(0)<B_{2}(0)$. Since bidder 1's expected payoff when valuation is zero is half the bid (bidder 1 loses with probability one), bidder 1 would be better off by increasing the bid up to $B_{2}(0)$. But this contradicts the assumption that $B_{1}(\cdot)$ is an equilibrium bidding strategy. Therefore $B_{1}(0)=B_{2}(0)$. Since bidders' expected payoff when their valuation is zero is half their bid, they will bid $a>0$ when their draw is zero. Consider now the terminal boundary condition. Assume, for contradiction, that $B_{1}(\bar{v})=b>\bar{v}$. Because of continuity, there exists $\theta$ such that $B_{1}(v)>\bar{v}$ for all $v \in[\theta, \bar{v}]$. 
Over this interval, partner 2 has a zero probability of winning when bidding the valuation. It is straightforward to prove that, to drive up the price that 1 has to pay for shares, bidder 2 will bid in equilibrium, $B_{2}(v)=$ $\lim _{\varepsilon \rightarrow 0} B_{1}(v)-\varepsilon$ for all $v \in[\theta, \bar{v}]$. This implies $U_{1}\left(\bar{v}, b, B_{2}\right)<U_{1}\left(\bar{v}, \bar{v}, B_{2}\right)$. Thus $B_{1}(\bar{v}) \leq \bar{v}$. The same argument applies to partner 2 , thus $B_{2}(\bar{v}) \leq \bar{v}$. Now $B_{i}(\bar{v})=\bar{v}$ for $i=1,2$, follows from standard auction theory since each partner will bid at least the valuation if there is a positive probability that the other one will bid at least that high.

Thus, by using an argument similar to those employed in Theorem 1, it can be shown that a pair of strategies $\left(B_{1}, B_{2}\right)$ is an equilibrium for the LBA if and only if the strategies are pure; the bid functions are strictly increasing and differentiable, with $B_{i}(v)>v$ for all $i$, and $v \in[0, \bar{v})$ and their inverse functions $\left(l_{1}(b), l_{2}(b)\right)$ are such that they are solutions to the system of differential equations

$$
2\left(l_{i}(b)-b\right) f_{j}\left(l_{j}(b)\right) w_{j}^{\prime}(b)+\left(1-F_{j}\left(l_{j}(b)\right)\right)=0,
$$

with boundary conditions $l_{1}(a)=l_{2}(a)=0$, and $l_{1}(\bar{v})=l_{2}(\bar{v})=\bar{v}$, where $a$ is a parameter belonging to $(0, \bar{v})$. We will now show that such an equilibrium exists and that under (A4) it is unique.

THEOREM 3. If assumptions (A1) and (A4) hold then there exists one and only one Bayesian equilibrium for the $L B A$.

Proof. The equations in system (2) of the equilibrium of the LBA can be rewritten as

$$
\frac{d}{d b} \ln \left(1-F_{j}\left(l_{j}(b)\right)\right)^{2}=\frac{1}{l_{i}(b)-b} \quad \text { for } i=1,2, \quad i \neq j .
$$

Consider the following change of variables $\widetilde{v}=\bar{v}-v$ and $\tilde{b}=\bar{v}-b$. Notice that this change of variables implies a change in the origin. With these new variables define the functions $\widetilde{l}_{i}(\widetilde{b}), i=1,2$, as follows:

$$
\tilde{l}_{i}(\tilde{b})=\bar{v}-l_{i}(\bar{v}-\tilde{b}) .
$$

The equations and boundary conditions that determine the equilibrium for the LBA become

$$
\begin{aligned}
\frac{d}{d \widetilde{b}} \ln \left(1-F_{j}\left(\bar{v}-\tilde{l}_{j}(\tilde{b})\right)\right)^{2} & =\frac{1}{\widetilde{l}_{i}(\widetilde{b})-\tilde{b}} \quad \text { for } i \neq j, \quad i=1,2 . \\
\tilde{l}_{1}(\widetilde{a}) & =\tilde{l}_{2}(\widetilde{a})=\bar{v}, \quad \text { where } \tilde{a} \in(0, \bar{v}),
\end{aligned}
$$

and

$$
\tilde{l}_{1}(0)=\tilde{l}_{2}(0)=0
$$


These equations and boundary conditions are identical to the equations and boundary conditions of the equilibrium of a first price auction with two bidders and valuation distributions $G_{1}$ and $G_{2}$ defined as follows:

$$
G_{i}(w)=\left(1-F_{i}(\bar{v}-w)\right)^{2} \quad \text { for } i=1,2 \text {, and } w \in[0, \bar{v}] .
$$

Existence and uniqueness follow immediately from Lebrun (1999). Notice that (A4) ensures that $(d / d v)\left(G_{1}(v) / G_{2}(v)\right)>0$ holds, and hence, uniqueness follows.

The following proposition shows some of the properties of the equilibrium induced by the loser bid auction.

Proposition 2. Under (A4) if $\left(B_{1}, B_{2}\right)$ is an equilibrium strategy profile to a loser-price auction then for all $i=1,2$,

(i) $B_{1}(v)>B_{2}(v)$, for all $v \in(0, \bar{v})$,

(ii) The distribution of bidder 2's bids stochastically dominates the distribution of bidder 1's bids.

Proof. We have already shown that the unique equilibrium of the LBA coincides with the unique equilibrium of a first price auction with two bidders and valuation distributions $G_{1}$ and $G_{2}$ defined as follows:

$$
G_{i}(w)=\left(1-F_{i}(\bar{v}-w)\right)^{2} \quad \text { for } i=1,2 \text {, and } w \in[0, \bar{v}] .
$$

From Maskin and Riley (1999) we know that $(d / d v)\left(G_{1}(v) / G_{2}(v)\right)>0$ implies $\tilde{l}_{1}(\tilde{b})>\tilde{l}_{2}(\tilde{b})$ and $G_{1}\left(\tilde{l}_{1}(\tilde{b})\right)<G_{2}\left(\tilde{l}_{2}(\tilde{b})\right)$ for all $\tilde{b} \in(0, \tilde{a})$. Because of (A4) we know that $(d / d v)\left(G_{1}(v) / G_{2}(v)\right)>0$ holds. Since $\tilde{b}_{i}=\bar{v}-b_{i}$ and $\tilde{l}_{i}(\tilde{b})=\bar{v}-l_{i}(\bar{v}-\tilde{b})$, we have that $l_{1}(b)<l_{2}(b)$ for all $b \in(a, \bar{v})$, consequently (i) follows. Finally, (ii) is deduced from $G_{1}\left(\tilde{l}_{1}(\tilde{b})\right)<G_{2}\left(\tilde{l}_{2}(\tilde{b})\right)$ that yields $F_{1}\left(l_{1}(b)\right)>F_{2}\left(l_{2}(b)\right)$ for all $b \in(a, \bar{v})$.

In the LBA, as in the WBA, the reputedly more interested bidder bids less aggressively. In both auctions bidder 2 has a higher probability of winning. Thus bidder 2 is better off in the auction that produces the lowest expected selling price. It seems reasonable to expect bidder 1 to have higher interim expected payoffs in a LBA than bidder 2. Notice that when she wins she has to pay a lower price for the assets whereas when losing she gets a higher price. In particular,

$U_{1}(\bar{v})=\int_{0}^{\bar{v}}\left(\bar{v}-0.5 B_{2}\left(v_{2}\right)\right) f_{2}\left(v_{2}\right) d v_{2}>\int_{0}^{\bar{v}}\left(\bar{v}-0.5 B_{1}\left(v_{1}\right)\right) f_{1}\left(v_{1}\right) d v_{1}=U_{2}(\bar{v})$

and $U_{1}(0)=U_{2}(0)=0.5 a$. Therefore either $U_{1}(v) \geq U_{2}(v)$ for all $v$, or at least this is the case for high valuations. Since in a LBA bidders overbid in equilibrium, the loser does not regret ex post to lose since a price is obtained for shares that is higher than the valuation for the asset. The 
winner, on the other hand, regrets ex post that they overbid so much but this overbidding is optimal ex ante. ${ }^{7}$

\subsubsection{Numerical Examples}

We have shown that both auction formats may yield to inefficient outcomes if the partners have valuations that are independent but asymmetrically distributed. If to guarantee ex post efficiency is the only goal of the partners then the following bidding procedure can be used. Each partner submits a bid and has to pay the expected externality associated with her bid; the asset is awarded to the partner making the highest bid. In our setup, partner $i$ submits $b_{i}$ and pays $p_{i}=\int_{0}^{b_{i}} v_{j} f_{j}\left(v_{j}\right) d v_{j}$ to the other partner. By internalizing the expected externality, this bidding scheme induces each partner to make an equilibrium bid equal to her valuation, $b_{i}=v_{i}$, which ensures an ex post efficient outcome. Note that this procedure has an important caveat since it imposes different prices on the partners. If both submit the same bid, partner 1 will have to pay a higher price to get the asset than 2 does. This price discrimination is hard to defend.

Our purpose here is to show through two particular numerical examples that the WBA can be more efficient whereas the LBA generates larger revenues (i.e., larger expected selling prices).

From the mechanism design literature, it is known that the expected total gains derived from the implementation of any of these auctions can be expressed as

$$
\begin{gathered}
\int_{0}^{\bar{v}} v_{1} P\left(B_{1}\left(v_{1}\right) \geq B_{2}\left(v_{2}\right)\right) d F_{1}\left(v_{1}\right)+\int_{0}^{\bar{v}} v_{2} P\left(B_{1}\left(v_{1}\right)<B_{2}\left(v_{2}\right)\right) d F_{2}\left(v_{2}\right) \\
=\int_{0}^{\bar{v}} v_{1} d F_{1}\left(v_{1}\right)+\int_{0}^{\bar{v}} \int_{0}^{\bar{v}}\left(v_{2}-v_{1}\right) m\left(v_{1}, v_{2}\right) d F_{1}\left(v_{1}\right) d F_{2}\left(v_{2}\right),
\end{gathered}
$$

where $m\left(v_{1}, v_{2}\right)=P\left(B_{1}\left(v_{1}\right)<B_{2}\left(v_{2}\right)\right)$.

Since an ex post efficient mechanism maximizes the expected total gains from trade, and since in such a mechanism

$$
m\left(v_{1}, v_{2}\right)= \begin{cases}1 & \text { if } v_{1}<v_{2} \\ 0 & \text { if } v_{1}>v_{2}\end{cases}
$$

then, on efficiency grounds, we seek for the auction that minimizes $\int_{\underline{b}}^{\bar{b}}\left(y_{2}(b)-y_{1}(b)\right) d b, y=w, l$.

Similarly, expected revenue can be expressed as

$$
R=\int_{0}^{\bar{v}} \int_{0}^{\bar{v}}\left[J_{1}\left(v_{1}\right) \pi_{1}\left(v_{1}, v_{2}\right)+J_{2}\left(v_{2}\right) \pi_{2}\left(v_{1}, v_{2}\right)\right] d F_{1}\left(v_{1}\right) d F_{2}\left(v_{2}\right)
$$

${ }^{7}$ Overbidding is also the optimal strategy in a takeover when bidders have partial ownership of the item and the valuations of the bidders are drawn from a common distribution. This overbidding may lead to inefficient outcomes. For an argument, see Burkart (1995). 
where $J_{i}(v)$ is the expected marginal revenue generated if the object is assigned to bidder $i$ (i.e., $J_{i}(v)=v-\left(1-F_{i}(v) / f_{i}(v)\right)$. Because of (A4) we have that $J_{1}(v)>J_{2}(v)$ for all $v \in(0, \bar{v})$. Thus expected total revenue is maximized by selling to the weak buyer, i.e., to bidder $1 .^{8}$

The purpose of this section is to compare the two auctions on efficiency and revenue grounds. To do so we rely on two particular numerical examples. These examples illustrate some of the features of the equilibrium induced by the WBA and the LBA.

EXAMPLE 1. Let $F_{1}(v)=(1-\exp (-v)) /(1-\exp (-1))$ and $F_{2}(v)=v$ with $v \in[0,1]$. Note that $F_{1}(v), F_{2}(v)$ satisfy all the assumptions in the model.

\section{The Winner Bid Auction}

The equilibrium bid functions are the solutions of the following system of differential equations:

$$
\begin{aligned}
2\left(w_{1}(b)-b\right) w_{2}^{\prime}(b) & =w_{2}(b), \\
2\left(w_{2}(b)-b\right)\left(\exp \left(-w_{1}(b)\right)\right) w_{1}^{\prime}(b) & =1-\exp \left(-w_{1}(b)\right), \\
w_{2}(0.62121) & =1, \\
w_{1}(0.62121) & =1 .
\end{aligned}
$$

There exists a simple algorithm to find a solution numerically. Pick any $\bar{b} \in(0, \bar{v})$ and compute the associated solution in system (1). If this solution intersects the 45 degree line below 0 , then increase the starting value. Otherwise, if $w_{i}(0)>0$, reduce the starting value. ${ }^{9}$

\section{The Loser Bid Auction}

The equilibrium bid functions are the solutions of the following system of differential equations:

$$
\begin{aligned}
2\left(l_{1}(b)-b\right) l_{2}^{\prime}(b) & =l_{2}(b)-1, \\
2\left(l_{2}(b)-b\right)\left(\exp \left(-l_{1}(b)\right)\right) l_{1}^{\prime}(b) & =\exp (-1)-\exp \left(-l_{1}(b)\right), \\
l_{2}(0.2869) & =0, \\
l_{1}(0.2869) & =0 .
\end{aligned}
$$

${ }^{8}$ This result is proved in Bulow and Roberts (1989). They showed that a revenue-maximizing auction allocates objects to the bidder(s) with the highest marginal revenue(s) rather than to those with the highest value(s). So a revenue-maximizing auctioneer discriminates in favor of selling to bidders whose values are drawn from lower distributions, that is, "weaker" bidders.

${ }^{9}$ For a study on how to solve numerically differential equations as those in (1), see Marshall et al. (1994). 
The algorithm to find a solution numerically works as follows: Pick any $a \in(0, \bar{v})$ and compute the associated solution in system (2). If this solution intersects the 45 degree line below $\bar{v}$, then decrease the starting value. Otherwise, if $w_{i}(\bar{v})>\bar{v}$, increase the starting value.

For the WBA, consider the function $W(v)$ implicitly defined by the equation

$$
w_{2}(b) \equiv W\left(w_{1}(b)\right)
$$

it is a mapping from $[0,1]$ onto $[0,1]$ with $W(v)>v$ for all $v \in(0,1)$. The function $W(v)$ is an "equilibrium correspondence:" in equilibrium, bidder 1 with valuation $v=w_{1}(b)$ and bidder 2 with valuation $W\left(w_{1}(b)\right)$ set the same bid. Notice, that if $W(v)=v$ for all $v$, then the WBA would yield efficient allocations.

Similarly, for the LBA, consider the function $L(v)$ implicitly defined by the equation

$$
l_{2}(b) \equiv L\left(l_{1}(b)\right)
$$

it is a mapping from $[0,1]$ onto $[0,1]$ with $L(v)>v$ for all $v \in(0,1)$.

Figure 1 depicts these two equilibrium correspondences. ${ }^{10}$

EXAMPLe 2. Assume now $F_{1}(v)=v$ and $F_{2}(v)=0.5\left(v+v^{2}\right)$ with $v \in$ $[0,1]$.

\section{The Winner Bid Auction}

The equilibrium bid functions are the solutions of the following system of differential equations:

$$
\begin{aligned}
\left(w_{1}(b)-b\right)\left(1+2 w_{2}(b)\right) w_{2}^{\prime}(b) & =0.5 w_{2}(b)\left(1+w_{2}(b)\right), \\
2\left(w_{2}(b)-b\right) w_{1}^{\prime}(b) & =w_{1}(b), \\
w_{2}(0.70337) & =1 \\
w_{1}(0.70337) & =1 .
\end{aligned}
$$

\footnotetext{
${ }^{10}$ For the LBA, we plot $v=l_{1}(b)$ versus $L\left(l_{1}(b)\right)$. Similarly, for the WBA, we plot $v=w_{1}(b)$ versus $W\left(w_{1}(b)\right)$. Note that we can plot both mappings in the same figure since, in both cases, the independent variable is $v$.
} 


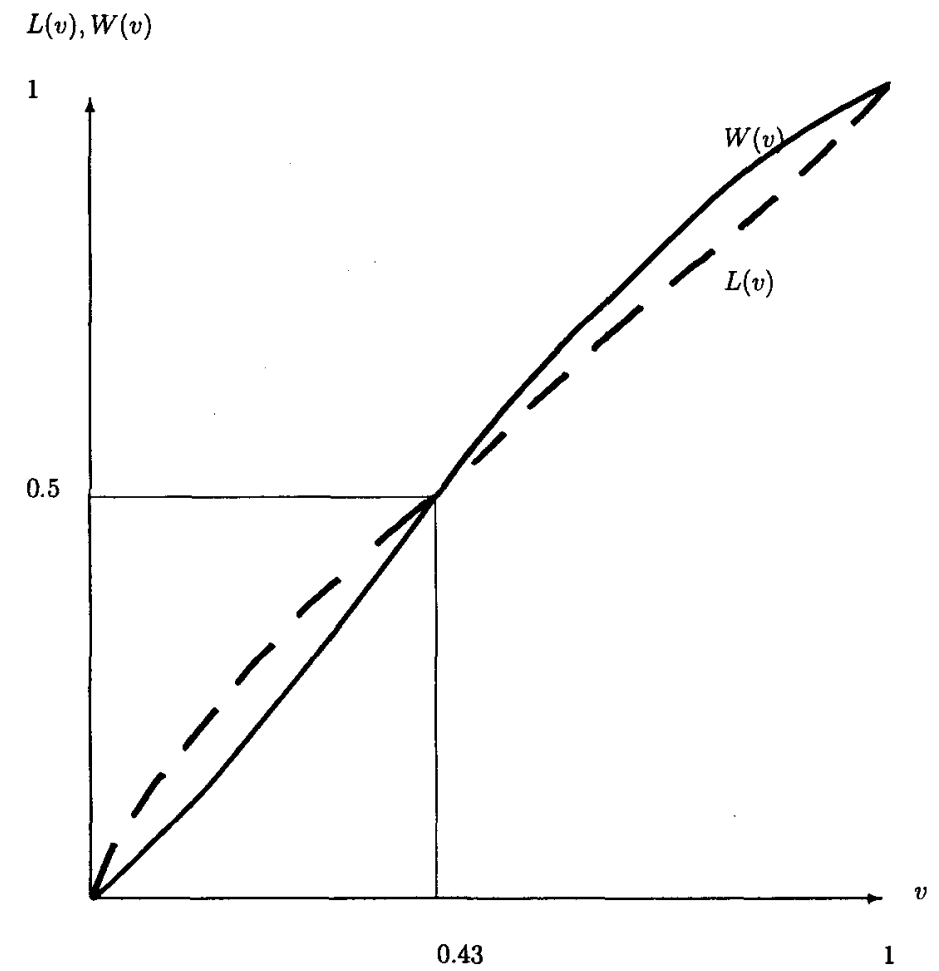

FIG. 1. Equilibrium correspondence: Example 1.

\section{The Loser Bid Auction}

The equilibrium bid functions are the solutions of the following system of differential equations:

$$
\begin{aligned}
\left(l_{1}(b)-b\right)\left(1+2 l_{2}(b)\right) l_{2}^{\prime}(b) & =0.5 l_{2}(b)\left(1+l_{2}(b)\right)-1, \\
2\left(l_{2}(b)-b\right) l_{1}^{\prime}(b) & =l_{1}(b)-1, \\
l_{2}(0.381271) & =0, \\
l_{1}(0.381271) & =0 .
\end{aligned}
$$

As in Example 1, let $W(v)$ be implicitly defined by the equation

$$
w_{2}(b) \equiv W\left(w_{1}(b)\right),
$$

and let $L(v)$ be implicitly defined by the equation

$$
l_{2}(b) \equiv L\left(l_{1}(b)\right) .
$$

As for Example 1, Fig. 2 depicts these two implicit mappings. 


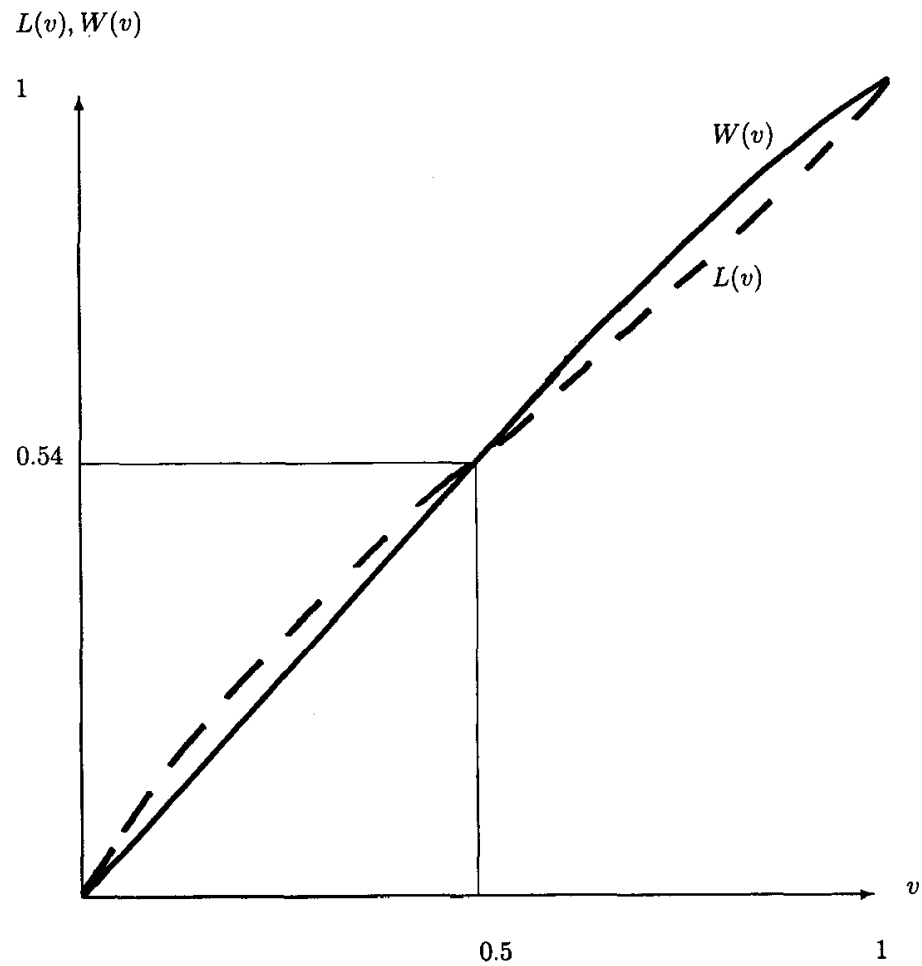

FIG. 2. Equilibrium correspondence: Example 2.

Results. The numerical results confirm that partner 2 bids more conservatively in all the auctions. In both examples it is satisfied that $w_{2}(b)>$ $w_{1}(b)$, and $l_{2}(v)>l_{1}(v)$ or equivalently, $W(v)>v$ and $L(v)>v$.

There exists $\widetilde{v}$ such that $W(\tilde{v})=L(\tilde{v})=0.5$. Moreover $W(v)<L(v)$ for all $v \in(0, \tilde{v})$ and $W(v)>L(v)$ for all $v \in(\tilde{v}, 1)$, with $L(0)=0=W(0)$ and $L(1)=1=W(1)$.

Under the LBA we obtain that both partners may overbid so that either winner loses money. In Example 1, for instance, if $v_{1}, v_{2} \in(0.3,0.85)$ either winner will suffer a loss.

Numerical computations show that the LBA generates, in both examples, a higher expected selling price than the WBA.

In Example 1, numerical computations, show that the WBA is more efficient. In Fig. 1, notice that $L(v)>W(v)>v$ for all $v_{1} \in(0,0.43), v_{2} \in$ $(0,0.5)$. Since bidder 1 is more likely to have valuations in the interval $(0,0.43)$ than in the interval $(0.43,1)$, being the intervals $(0,0.5)$ and $(0.5,1)$ equally likely for bidder 2 , the ex-ante probability of inefficient outcomes is larger in the LBA. In Example 2, numerical computations show 
that the LBA is more efficient. In Fig. 2, notice that $W(v)>L(v)>v$ for all $v_{1} \in(0.5,1), v_{2} \in(0.54,1)$. Since bidder 2 is more likely to have valuations in the interval $(0.54,1)$ than in the interval $(0,0.54)$, being the intervals $(0,0.5)$ and $(0.5,1)$ equally likely for bidder 1 , the ex ante probability of inefficient outcomes is larger in the WBA.

\section{REVENUE COMPARISONS}

\subsection{The Symmetric Case}

Let us assume that partners' valuations are independent and identically distributed from a cumulative distribution function $F$ with support $[0,1]$ and positive continuous density function $f$. Under these assumptions it is known that the equilibrium bid for the WBA is

$$
B_{i}^{w}(v)=v-\frac{\int_{0}^{v}[F(z)]^{2} d z}{[F(v)]^{2}} \quad \text { for } i=1,2,
$$

whereas the equilibrium bid for the LBA is

$$
B_{i}^{l}(v)=v-\frac{\int_{1}^{v}[F(z)-1]^{2} d z}{[F(v)-1]^{2}} \quad \text { for } i=1,2 .
$$

The expected selling price in the WBA will be $P^{w}=\int_{0}^{1} B^{w}(v) F(v) f(v) d v$ and in the LBA will be $P^{l}=\int_{0}^{1}\left(\int_{0}^{v} B^{L}(z) f(z) d z\right) f(v) d v$.

PROPOSITION 3. If both partners valuations are distributed according to the same c.d.f. F, then the expected selling price is strictly greater in a $L B A$ than in a $W B A .^{11}$

Proof. Substituting the equilibrium bids into the expressions for the expected selling price, and integrating by parts, yields

$$
\begin{aligned}
P^{l} & =\int_{0}^{1}\left[\int_{0}^{v} z f(z) d z\right] d F(v)+\int_{0}^{1}\left[\int_{0}^{v} \frac{f(t) \int_{t}^{1}[F(z)-1]^{2} d z}{[F(t)-1]^{2}} d t\right] d F(v) \\
& =\left|F(v) \int_{0}^{v} z f(z) d z\right|_{0}^{1}-\int_{0}^{1} v f(v) F(v) d v
\end{aligned}
$$

${ }^{11}$ For uniform distribution functions, Singh (1995) obtains this result for the pure privatevalues case, and Bulow et al. (1999) for the common-values case. Our contribution here is to show that in the private-values case the result holds for any continuous and differentiable distribution function. 


$$
\begin{aligned}
& +\int_{0}^{1}\left[\left|(1-F(t))^{-1} \int_{t}^{1}[F(z)-1]^{2} d z\right|_{0}^{v}-\int_{0}^{v}(F(t)-1) d t\right] d F(v) \\
= & \int_{0}^{1} v f(v) d v-\int_{0}^{1} v f(v) F(v) d v \\
& +\int_{0}^{1}\left[(1-F(v))^{-1} \int_{v}^{1}[F(z)-1]^{2} d z\right] d F(v) \\
& -\int_{0}^{1}[1-F(v)]^{2} d v+\int_{0}^{1}\left(\int_{0}^{v}(1-F(t)) d t\right) d F(v) \\
= & \int_{0}^{1} v f(v) d v-\int_{0}^{1} v f(v) F(v) d v-\left|\ln (1-F(v)) \int_{v}^{1}[F(z)-1]^{2} d z\right|_{0}^{1} \\
& -\int_{0}^{1} \ln (1-F(v))[1-F(v)]^{2} d v-\int_{0}^{1}[1-F(v)]^{2} d v \\
& +\left|F(v) \int_{0}^{v}(1-F(z)) d z\right|_{0}^{1}-\int_{0}^{1}(1-F(v)) F(v) d v \\
= & \int_{0}^{1} v f(v)(1-F(v)) d v-\int_{0}^{1} \ln (1-F(v))[1-F(v)]^{2} d v \\
= & \int_{0}^{1}[1-F(v)]^{2}[0.5-\ln (1-F(v))] d v,
\end{aligned}
$$

and

$$
\begin{aligned}
P^{w} & =\int_{0}^{1} v F(v) f(v) d v-\int_{0}^{1} \frac{f(v)}{F(v)}\left(\int_{0}^{v} F^{2}(z) d z\right) d v \\
& =0.5-\int_{0}^{1} F^{2}(v)[0.5-\ln F(v)] d v
\end{aligned}
$$

Therefore

$$
\begin{aligned}
2\left(P^{l}-P^{w}\right)= & \int_{0}^{1}\left([1-F(v)]^{2}[1-2 \ln (1-F(v))]\right. \\
& \left.+F^{2}(v)[1-2 \ln F(v)]\right) d v-1
\end{aligned}
$$

Let us define

$$
H(v)=[1-v]^{2}\left[1-\ln (1-v)^{2}\right]+v^{2}[0.5-\ln v] .
$$

It is straightforward to see that $H(v)$ is strictly larger than 1 for all $v \in$ $(0,1)$ with $\lim _{v \rightarrow 0} H(v)=1$ and $\lim _{v \rightarrow 1} H(v)=1$. Moreover, $H^{\prime}(v)=$ $4[(1-v) \ln (1-v)-v \ln v]$ with $\lim _{v \rightarrow 0} H^{\prime}(v)=0$ and $\lim _{v \rightarrow 1} H^{\prime}(v)=1$. Now, $H(v) \geq 1$ for all $v \in[0,1]$ implies $H(F(v)) \geq 1$ for all $v \in[0,1]$ since $F(v) \in[0,1]$. Therefore $\int_{0}^{1} H(F(v)) d v \geq 1$, and, consequently, $0 \leq$ $\int_{0}^{1}(H(F(v))-1) d v=P^{l}-P^{w}$. 
Remark 1. If $F_{2}$ dominates $F_{1}$ in the sense of (A4) then the expected selling price in a LBA, when both bidders have valuations drawn form $F_{1}$, is smaller than when valuations are drawn from $F_{2}$. Similarly, if $F_{2}$ dominates $F_{1}$ in the sense of (A3), then the expected selling price in a WBA, when both bidders have valuations drawn form $F_{1}$, is smaller than when valuations are drawn from $F_{2}$.

In the symmetric case the LBA outperforms the WBA. McAfee (1992) has shown that both of them are efficient and yield the same interim expected utility to the bidders. Here we have shown that the LBA generates larger expected prices. Thus, based on revenue considerations, the LBA should be chosen over the WBA. Recall that this procedure is commonly used in takeovers whereas the WBA is rarely used.

\subsection{Asymmetric Versus Symmetric Auctions}

We will now compare bidding in the asymmetric auctions with bidding when both buyers valuations are distributed according to the same cumulative distribution. To do so, for the WBA, we will denote by $B w_{i}^{A}(v)$ to the equilibrium bid function of bidder $i$ in the asymmetric auction, and by $B w_{i}^{S}(v)$ to the symmetric equilibrium bid function when both buyers valuations are drawn from $F_{i}(\cdot)$. Similarly, for the LBA, we will denote by $B l_{i}^{A}(v)$ to the equilibrium bid function of bidder $i$ in the asymmetric case, and by $B l_{i}^{S}(v)$ to the equilibrium bid function in the symmetric case. For the expected selling price we will use the following notation: $P_{A}^{w}$ and $P_{A}^{l}$ denote the expected selling price in the asymmetric WBA and LBA, respectively; $P_{S i}^{w}$ stands for the expected selling price in a symmetric WBA when both buyers valuations are drawn from $F_{i}(\cdot)$, and similarly for $P_{S i}^{l}$.

PROPOSITION 4. If (A3) holds then

(i) $B w_{1}^{A}(v)>B w_{1}^{S}(v)$ for all $v \in(0, \bar{v})$ with $P_{A}^{w}>P_{S 1}^{w}$.

(ii) $B w_{2}^{A}(v)<B w_{2}^{S}(v)$ for all $v \in(0, \bar{v})$ with $P_{A}^{w}<P_{S 2}^{w}$.

If (A4) holds then

(iii) $B l_{1}^{A}(v)>B l_{1}^{S}(v)$ for all $v \in(0, \bar{v})$ with $P_{A}^{l}>P_{S 1}^{l}$.

(iv) $B l_{2}^{A}(v)<B l_{2}^{S}(v)$ for all $v \in(0, \bar{v})$ with $P_{A}^{l}<P_{S 2}^{l}$.

Proof. See appendix.

Proposition 4 shows that, irrespective of the auction format considered, the expected price in the asymmetric auction is always larger than the expected selling price when both bidders are weak (both bidders valuations are drawn from $F_{1}$ ), but it is smaller than the expected selling price when both bidders are strong (both bidders valuations are drawn from $F_{2}$ ). Therefore, if the expected price in the LBA with two weak bidders is higher than 
the expected price in the WBA with two strong bidders, we can conclude that the expected price in the asymmetric LBA will be larger than in the asymmetric WBA. This is the case, for instance, if the weak bidders have valuations drawn from a uniform distribution and the strong bidders have valuations drawn from a distribution $F_{2}(v)=v^{a}$ for any $a \in(1,1.2655)$.

\subsection{Comparison of Auction Procedures}

Proposition 5. The expected selling price in a $L B A$ when valuations are drawn from $F_{1}(v), F_{2}(v)$ is equal to one minus the expected selling price in a WBA with valuations drawn from $\left(1-F_{1}(1-v), 1-F_{2}(1-v)\right)$ for $v \in$ $[0,1]$.

Proof. Consider first a WBA. If bidder 1 with valuation $w_{1}(b)$ bids $b$ his interim expected payment will be $b F_{2}\left(w_{2}(b)\right)$. The ex ante expected payment of this bidder will be

$$
P_{1}^{w}=\int_{0}^{b^{*}} b F_{2}\left(w_{2}(b)\right) d F_{1}\left(w_{1}(b)\right) .
$$

Similarly, if bidder 2 with valuation $w_{2}(b)$ bids $b$ her interim expected payment will be $b F_{1}\left(w_{1}(b)\right)$. The ex ante expected payment of this bidder will be

$$
P_{2}^{w}=\int_{0}^{b^{*}} b F_{1}\left(w_{1}(b)\right) d F_{2}\left(w_{2}(b)\right) .
$$

The expected price in a $W B A, P^{w}=P_{1}^{w}+P_{2}^{w}$.

Consider now a LBA. If bidder 1 with valuation $l_{1}(z)$ bids $z$ her interim expected revenue will be $z\left(1-F_{2}\left(l_{2}(z)\right)\right)$, i.e., the revenue she expects to get is the price paid by bidder 2 when bidder 2 wins. The ex ante expected revenue of bidder 1 will be

$$
R_{1}^{l}=\int_{a}^{1} z\left(1-F_{2}\left(l_{2}(z)\right)\right) d l_{1}(z) .
$$

Similarly,

$$
R_{2}^{l}=\int_{a}^{1} z\left(1-F_{1}\left(l_{1}(z)\right) d F_{2}\left(l_{2}(z)\right) .\right.
$$

The expected selling price in a $L B A$ will be $P^{l}=R_{1}^{l}+R_{2}^{l}$.

Since $l_{i}(b)=1-r_{i}(1-b)$ for $i=1,2$, where $r_{i}(\cdot)$ denotes the inverse bidding function of bidder $i$ in a WBA when valuations are drawn from $1-F_{1}(1-w), 1-F_{2}(1-w)$ (see proof of Theorem 3), we have that

$$
\begin{aligned}
P^{w}= & \int_{a}^{1} z\left(1-F_{2}\left(1-r_{2}(1-z)\right)\right) d F_{1}\left(1-r_{1}(1-z)\right) \\
& +\int_{a}^{1} z\left(1-F_{1}\left(1-r_{1}(1-z)\right) d F_{2}\left(1-r_{2}(1-z)\right) .\right.
\end{aligned}
$$


By making a change of variables, $(b=1-z)$, and integrating by parts, we obtain

$$
\begin{aligned}
P^{l}= & \int_{0}^{b^{* *}}(1-b)\left(1-F_{2}\left(1-r_{2}(b)\right)\right) f_{1}\left(1-r_{1}(b)\right) d r_{1}(b) \\
& +\int_{0}^{b^{* *}}(1-b)\left(1-F_{1}\left(1-r_{1}(b)\right) f_{2}\left(1-r_{2}(b)\right) d r_{2}(b)\right. \\
= & \int_{0}^{b^{* *}}\left(1-F_{2}\left(1-r_{2}(b)\right)\right) f_{1}\left(1-r_{1}(b)\right) d r_{1}(b) \\
& +\int_{0}^{b^{* *}}\left(1-F_{1}\left(1-r_{1}(b)\right) f_{2}\left(1-r_{2}(b)\right) d r_{2}(b)-P_{J_{1}, J_{2}}^{w}\right. \\
= & \left|\left(1-F_{2}\left(1-r_{2}(b)\right)\right)\left(1-F_{1}\left(1-r_{1}(b)\right)\right)\right|_{0}^{b^{* *}}-P_{J_{1}, J_{2}}^{w} \\
= & 1-P_{J_{1}, J_{2}}^{w},
\end{aligned}
$$

where $P_{J_{1}, J_{2}}^{w}$ denotes the expected selling price in a WBA with valuations drawn from $\left(J_{1}, J_{2}\right)=\left(1-F_{1}(1-w), 1-F_{2}(1-w)\right)$, and where $b^{* *}=$ $1-a$.

Proposition 6. (i) If $F_{1}(v)=v$ and $F_{2}(\cdot)$ is a convex function then $P^{l}>1-P^{w}$. Moreover, a sufficient condition for the expected selling price in a $L B A$ to be larger than in a WBA is $P_{S 1}^{w}+P_{S 2}^{w}<1$.

(ii) If $F_{2}(v)=v$ and $F_{1}(\cdot)$ is a concave function then $P^{l}<1-P^{w}$. Moreover, a sufficient condition for the expected selling price in a $L B A$ to be larger than in a WBA is $P_{S 1^{\prime}}^{w}+P_{S 2}^{w}<1$, where $P_{S 1^{\prime}}^{w}$ denotes the expected selling price in a symmetric WBA when both bidders valuations are drawn from $G_{1}(v)=1-F_{1}(1-v)$.

Proof. (i) The result follows from Theorem 1 in Lebrun (1998) and the result in previous proposition. Lebrun has shown that if $\left(J_{1}, J_{2}\right)=\left(F_{1}, F_{2}\right)$, and $\left(J_{1}, J_{2}^{\prime}\right)=\left(F_{1}, 1-F_{2}(\bar{v}-w)\right)$ (notice that the convexity of $F_{2}$ implies $\left.J_{2}>J_{2}^{\prime}\right)$ then $P_{J_{1}, J_{2}}^{w}>P_{J_{1}, J_{2}^{\prime}}^{w}{ }^{12}$ From Proposition 5 we know that $P^{l}=$ $1-P_{J_{1}, J_{2}^{\prime}}^{w}$. Thus $P^{l}>1-P^{w}$. Moreover $P^{l}>P^{w}$ if $1>P_{J_{1}, J_{2}}^{w}+P_{J_{1}, J_{2}^{\prime}}^{w}$. Since $P_{J_{1}, J_{2}^{\prime}}^{w}<P_{J_{1}, J_{1}}^{w}$ and $P_{J_{1}, J_{2}}^{w}<P_{J_{2}, J_{2}}^{w}$ we get our first sufficient condition. To show (ii) we first notice that if $\left(J_{1}, J_{2}\right)=\left(F_{1}, F_{2}\right)$, and $\left(J_{1}^{\prime}, J_{2}\right)=(1-$ $\left.F_{1}(\bar{v}-w), F_{2}\right)$ then the concavity of $F_{1}$ implies $J_{1}^{\prime} \succ J_{1}$ and consequently

\footnotetext{
${ }^{12} \mathrm{He}$ shows these results for asymmetric first price auctions. We can apply them to the WBA by appealing to the results in Theorem 2 .
} 
$P_{J_{1}^{\prime}, J_{2}}^{w}>P_{J_{1}, J_{2}}^{w}$. Thus $P^{l}<1-P^{w}$. Now $P^{l}>P^{w}$ if $1>P_{J_{1}, J_{2}}^{w}+P_{J_{1}^{\prime}, J_{2}}^{w}$. Since $P_{J_{1}^{\prime}, J_{2}}^{w}<P_{J_{1}^{\prime}, J_{1}^{\prime}}^{w}$ and $P_{J_{1}, J_{2}}^{w}<P_{J_{2}, J_{2}}^{w}$ we get the second sufficient condition.

Remark 2. In the first numerical example considered in the paper $P_{S 1^{\prime}}^{w}=$ 0.341 and $P_{S 2}^{w}=0.444$ thus $P^{l}>P^{w}$. In the second numerical example $P_{S 1}^{w}=0.444$ and $P_{S 2}^{w}=0.35$ thus $P^{l}>P^{w}$.

\section{CONCLUSIONS}

It is well-known that private-value auctions with equal ownership of the item yield efficient outcomes when the valuations of the bidders are drawn from a common distribution. This result, as shown in this paper, does not carry out to the case of asymmetric distributions. The inefficiency is due to the fact that the bidder who is reputed to be more interested in the asset to be traded will bid less aggressively in equilibrium. As a result of this, the bidder with the lower valuation buys the target with positive probability. In both auctions the strong bidder has a higher probability of winning whereas a revenue-maximizing auctioneer would prefer weak bidders to win.

Results in this paper have a wide range of applications including the sharing of profits in bidding rings or creditors' bidding in Bankruptcy auctions. However, a limitation of the analysis in this paper is that attention is restricted to the special case of equal shares in the partnership or, in the takeover model, to symmetric toeholds.

\section{APPENDIX}

Proof of Lemma 1. To see that equilibrium strategies, $\left(B_{1}, B_{2}\right)$, have to be monotone increasing on $[0, \bar{v}]$ let $b^{\prime}=B_{i}\left(v_{i}^{\prime}\right)$ and $b^{\prime \prime}=B_{i}\left(v_{i}^{\prime \prime}\right)$ with $v_{i}^{\prime} \geq v_{i}^{\prime \prime}$. Equilibrium requires the following two conditions to be satisfied: $U_{i}\left(v_{i}^{\prime}, b^{\prime}, B_{j}\right) \geq U_{i}\left(v_{i}^{\prime}, b^{\prime \prime}, B_{j}\right)$, and $U_{i}\left(v_{i}^{\prime \prime}, b^{\prime \prime}, B_{j}\right) \geq U_{i}\left(v_{i}^{\prime \prime}, b^{\prime}, B_{j}\right)$, i.e.,

$$
\begin{aligned}
& \left(2 v_{i}^{\prime}-b^{\prime}\right) F_{j}\left(w_{j}\left(b^{\prime}\right)\right)+\int_{w_{j}\left(b^{\prime}\right)}^{\bar{v}} B_{j}(x) d F_{j}(x) \\
& \quad \geq\left(2 v_{i}^{\prime}-b^{\prime \prime}\right) F_{j}\left(w_{j}\left(b^{\prime \prime}\right)\right)+\int_{w_{j}\left(b^{\prime \prime}\right)}^{\bar{v}} B_{j}(x) d F_{j}(x)
\end{aligned}
$$


and

$$
\begin{aligned}
& \left(2 v_{i}^{\prime \prime}-b^{\prime \prime}\right) F_{j}\left(w_{j}\left(b^{\prime \prime}\right)\right)+\int_{w_{j}\left(b^{\prime \prime}\right)}^{\bar{v}} B_{j}(x) d F_{j}(x) \\
& \geq\left(2 v_{i}^{\prime \prime}-b^{\prime}\right) F_{j}\left(w_{j}\left(b^{\prime}\right)\right)+\int_{w_{j}\left(b^{\prime}\right)}^{\bar{v}} B_{j}(x) d F_{j}(x) .
\end{aligned}
$$

Subtracting the right-hand side of the second inequality from the lefthand side of the first, and subtracting the left-hand side of the second inequality from the right-hand side of the first, yields $\left(v_{i}^{\prime \prime}-v_{i}^{\prime}\right)\left[F_{j}\left(w_{j}\left(b^{\prime \prime}\right)\right)-\right.$ $\left.F_{j}\left(w_{j}\left(b^{\prime}\right)\right)\right] \geq 0$. This implies $b^{\prime \prime} \leq b^{\prime}$. Moreover, $B_{i}(\cdot)$ must be gapless: if there is a gap $\left[b^{\prime}, b\right]$ in $B_{i}$ then there must be a gap $\left(b^{\prime}, b\right)$ in $B_{j}$, because for any $v_{j}$ it would be better to bid $b^{\prime}$ than any other bid in that interval (since with this bid bidder $j$ lowers the price if winning without affecting the probability of winning). But then the type of bidder $i$ planning to bid $b$ would be better off bidding $\left(b^{\prime}+b\right) / 2$. Furthermore, $B_{i}$ must be atomless. Suppose not, then there is $b$ and $j$ such that $P\left(B_{j}\left(v_{j}\right)=b\right)>0$. Then there exists $\varepsilon>0$ such that bidder $i$ will assign zero probability to ( $b-$ $\varepsilon, b)$ creating a gap. Equilibrium strategies are strictly increasing since they are atomless and weakly monotonically increasing; and because they are also gapless then equilibrium has to be in pure strategies. Continuity and monotonicity of $B_{i}(\cdot)$ imply that $B_{i}(\cdot)$ is differentiable almost everywhere on its domain.

Consider now the boundary conditions. We first show that for a bidder with valuation $v$ a bid strictly larger than $v$ and which has a strictly positive probability of winning is less advantageous than a bid of $v$ and will thus not be played at the equilibrium, i.e., $U_{i}\left(v, v, B_{j}\right)-U_{i}\left(v, v+\varepsilon, B_{j}\right)>0$. Clearly,

$$
\begin{aligned}
& 0.5\left(v F_{j}\left(B_{j}^{-1}(v)\right)+\int_{B_{j}^{-1}(v)}^{\bar{v}} B_{j}(x) d F_{j}(x)-(v-\varepsilon) F_{j}\left(B_{j}^{-1}(v+\varepsilon)\right)\right. \\
& \left.\quad-\int_{B_{j}^{-1}(v+\varepsilon)}^{\bar{v}} B_{j}(x) d F_{j}(x)\right) \\
& =0.5\left(v\left(F_{j}\left(B_{j}^{-1}(v)\right)-F_{j}\left(B_{j}^{-1}(v+\varepsilon)\right)\right)+\varepsilon F_{j}\left(B_{j}^{-1}(v+\varepsilon)\right)\right. \\
& \left.\quad+\int_{B_{j}^{-1}(v)}^{B_{j}^{-1}(v+\varepsilon)} B_{j}(x) d F_{j}(x)\right) \\
& =0.5\left(\varepsilon F_{j}\left(B_{j}^{-1}(v+\varepsilon)\right)+\int_{B_{j}^{-1}(v)}^{B_{j}^{-1}(v+\varepsilon)}\left(B_{j}(x)-v\right) d F_{j}(x)\right)>0 .
\end{aligned}
$$


Hence, $B_{i}(v) \leq v$, for $i=1,2$, and, consequently, $B_{i}(0) \leq 0$. Now, if $B_{j}(0)<B_{i}(0)$ then, by continuity, there exists $\tilde{v}>0$ such that $B_{j}(\tilde{v})<$ $B_{i}(0) \leq 0$. Since type $\tilde{v}$ of partner $j$ is selling with probability one and gets a non-positive price, this type would do better by increasing the bid to 0 , paying zero price when winning and receiving a non-negative price when losing. Therefore $B_{1}(0)=B_{2}(0)=0$. Consider now the upper support of the bids distribution. Since $B_{1}(\cdot)$ is strictly increasing, bidder 2 wins with probability 1 by bidding $B_{1}(\bar{v})$. Hence $B_{2}(\bar{v}) \leq B_{1}(\bar{v})$. The same argument holds for bidder 1; thus, we also have $B_{1}(\bar{v}) \leq B_{2}(\bar{v})$, and hence $B_{1}(\bar{v})=B_{2}(\bar{v})$ $=\bar{b} \leq v$. If $\bar{b}=\bar{v}$ then type $\bar{v}$ of bidder 1 (the same argument applies for bidder 2) is making zero profits. By decreasing the bid by $\varepsilon$ they gain $0.5 \varepsilon$ when they still buy (which happens with a probability close to 1 ), and they lose less than $0.5 \varepsilon$ when they end up selling (which happens with a probability that can be made arbitrarily small by reducing $\varepsilon$ ). Thus $B_{1}(\bar{v})=B_{2}(\bar{v})=\bar{b}<v$.

Proof of Theorem 1. Let $B_{i}(\cdot)$ denote an equilibrium strategy for bidder $i$. The fact that $B_{i}(\cdot)$ is strictly increasing and differentiable almost everywhere on $[0, \bar{v}]$ implies that it has an inverse function $w_{i}$ defined on $[0, \bar{b}]$ which is increasing and differentiable almost everywhere and satisfies that $w_{i}(0)=0$ and $w_{i}(\bar{b})=\bar{v}$ for all $i=1,2$. Thus for all $i=1,2$, $U_{i}\left(v_{i}, B_{i}, B_{j}\right)$ is differentiable in its second argument. Hence $B_{i}(\cdot)$ must satisfy the first order condition for a maximum and therefore its inverse will satisfy system (1).

We will now show that the necessary conditions are sufficient. Assume $w_{1}, w_{2}$ verify (1), and satisfy the boundary conditions with $0<w_{i}(b)<\bar{v}$ for all $i=1,2$ and $b \in(0, \bar{b}]$. Note that $w_{i}^{\prime}(b)>0$ for all $i$ and $b \in(0, \bar{b}]$. If there exists $i$ and $\theta \in(0, \bar{b}]$ such that $w_{i}^{\prime}(\theta)=0$, then system (1) implies that $w_{j}(\theta)=\infty$ in contradiction with $0<w_{j}(b)<\bar{v}$, for $b \in(0, \bar{b}]$. Similarly, if there exists $\pi \in(0, \bar{b}]$, such that $w_{i}^{\prime}(\pi)<0$, then the boundary condition $w_{i}(0)=0$ and the fact that $w_{i}(b)>0$ for $b>0$ imply that there exists $\xi \in(0, \pi)$ satisfying $w_{i}^{\prime}(\xi)=0$. But this yields a contradiction with $0<w_{j}(b)<\bar{v}$ for $b \in(0, \bar{b}]$. Therefore $w_{1}, w_{2}$ are continuous, differentiable over $(0, \bar{b}]$ with $w_{i}(b)>b$, for all $b \in(0, \bar{b}]$ and all $i=1,2$. We have to show that $B_{1}=w_{1}^{-1}$ and $B_{2}=w_{2}^{-1}$ is an equilibrium. To do so, let us assume that partner 2 follows the strategy $B_{2}=w_{2}^{-1}$. Clearly, a bid larger than $\bar{b}$ is never a best response.

If $v_{1}=0$, then the best response is $B_{1}(0)=0$ because $b=0$ maximizes $U_{1}$ for $v_{1}=0$. Recall that

$$
U_{1}\left(0, b, B_{2}\right)= \begin{cases}\int_{0}^{\bar{v}} 0.5 B_{2}\left(v_{2}\right) d F_{2}\left(v_{2}\right) & \text { if } b=0, \\ -b F_{2}\left(B_{2}^{-1}(b)\right)+\int_{B_{2}^{-1}(b)}^{\bar{v}} 0.5 B_{2}\left(v_{2}\right) d F_{2}\left(v_{2}\right) & \text { if } b>0 .\end{cases}
$$


If $v_{1} \in(0, \vec{v})$ then we have

$$
\frac{\partial U_{1}\left(v_{1}, b, B_{2}\right)}{\partial b}=-\frac{1}{2} F_{2}\left(w_{2}(b)\right)+\left(v_{1}-b\right) f_{2}\left(w_{2}(b)\right) w_{2}^{\prime}(b) .
$$

Since $w_{2}^{\prime}(b)$ satisfies $(1)$ then

$$
\frac{\partial U_{1}\left(v_{1}, b, B_{2}\right)}{\partial b}=-\frac{1}{2} F_{2}\left(w_{2}(b)\right)+\frac{v_{1}-b}{w_{1}(b)-b} \cdot \frac{1}{2} F_{2}\left(w_{2}(b)\right) .
$$

Because $w_{1}(b)>b, \partial U_{1} / \partial b$ is positive if $v_{1}>w_{1}(b)$ and negative if $v_{1}<$ $w_{1}(b)$. For $v_{1} \in(0, \bar{v})$, the continuity of $U_{1}$ as a function of $b$ and the above results imply that $U_{1}$ is monotonically non-decreasing on $\left[0, B_{1}\left(v_{1}\right)\right.$ ) and monotonically non-increasing on $\left(B_{1}\left(v_{1}\right), \infty\right)$.

Therefore bidder 1's expected payoff is maximized if $v_{1}=w_{1}(b)$ that is if $b_{1}=B_{1}\left(v_{1}\right)$. Thus $B_{1}\left(v_{1}\right)$ is a best response if bidder 2 bids according with $B_{2}\left(v_{2}\right)$. Since a symmetric argument applies for bidder 2 , any strictly increasing and differentiable $w_{1}(b), w_{2}(b)$ satisfying system (1) and the boundary conditions define an equilibrium.

Proof of Proposition 4. The proposition follows from Theorem 1 and Corollary 1 in Lebrun (1998). This theorem establishes the following: Take $J_{1}, J_{2}, J_{2}^{\prime}$ such that $J_{2}^{\prime}$ dominates stochastically in the sense of reverse hazard rate to $J_{2}$ (using Lebrun's notation $J_{2}^{\prime} \succ J_{2}$ ). Assume that $J_{1}(0)=$ $J_{2}(0)=J_{2}^{\prime}(0)=0$ and $J_{2} \succ J_{1}$ or $J_{1} \succ J_{2}$, and $J_{2}^{\prime} \succ J_{1}$ or $J_{1} \succ J_{2}^{\prime}$. Let the bid functions and their inverses at the unique equilibrium when valuations are $\left(J_{1}, J_{2}\right)$ be denoted by $\left(B_{1}, B_{2}\right)$ and $\left(\alpha_{1}, \alpha_{2}\right)$, with $P$ denoting the corresponding expected selling price, and, at the unique equilibrium when the distributions are $\left(J_{1}, J_{2}^{\prime}\right)$ be denoted by $\left(B_{1}^{\prime}, B_{2}^{\prime}\right)$ and $\left(\alpha_{1}^{\prime}, \alpha_{2}^{\prime}\right)$, respectively, with $P^{\prime}$ denoting the corresponding expected selling price. Under these assumptions, it is shown that in a first-price auction $B_{1}^{\prime}(v)>B_{1}(v)$ for all $v \in(0, \bar{v}), J_{2}^{\prime}\left(\alpha_{2}^{\prime}(b)\right)<J_{2}\left(\alpha_{2}(b)\right)$ for all $b \in\left(0, B_{1}(\bar{v})=B_{2}(\bar{v})\right)$ and $P^{\prime}>P$. To show (i) and (ii) we will use the fact that the equilibrium bidding functions in a WBA when the distributions are $\left(F_{1}, F_{2}\right)$ coincide with the equilibrium bidding functions in a first price auction when the distributions are $\left(F_{1}^{2}, F_{2}^{2}\right)$. Therefore in (i) we have $\left(J_{1}, J_{2}\right)=\left(F_{1}^{2}, F_{1}^{2}\right)$ and $\left(J_{1}, J_{2}^{\prime}\right)=\left(F_{1}^{2}, F_{2}^{2}\right)$, and the result follows from the fact that $F_{2} \succ F_{1}$. In (ii) we have $\left(J_{1}, J_{2}\right)=\left(F_{2}^{2}, F_{1}^{2}\right)$ and $\left(J_{1}, J_{2}^{\prime}\right)=\left(F_{2}^{2}, F_{2}^{2}\right)$ and the result follows from the fact that $F_{2} \succ F_{1}$. To show (iii) and (iv) we first recall that by making a change of variables $\left(\tilde{l}_{i}(\tilde{b})=\bar{v}-l_{i}(\bar{v}-\tilde{b})\right)$ the equilibrium bidding functions in a LBA when the distributions are $\left(F_{1}, F_{2}\right)$ coincide with the equilibrium bidding functions in a first price auction when the distributions are $\left(G_{1}(w), G_{2}(w)\right)=\left(\left(1-F_{1}(\bar{v}-w)\right)^{2},\left(1-F_{2}(\bar{v}-w)\right)^{2}\right)$ for $w \in[0, \bar{v}]$. Because of $(\mathrm{A} 4), G_{1}(w) \succ G_{2}(w)$. Thus, in (iii) we have $\left(J_{1}, J_{2}\right)=\left(G_{1}, G_{2}\right)$ and $\left(J_{1}, J_{2}^{\prime}\right)=\left(G_{1}, G_{1}\right)$. Therefore $\tilde{l}_{1}^{\prime}\left(\tilde{b}^{\prime}\right)<\tilde{l}_{1}(\tilde{b})$ and 
$G_{1}\left(\widetilde{l}_{1}^{\prime}(\tilde{b})\right)<G_{2}\left(\widetilde{l}_{2}(\tilde{b})\right)$ implying $l_{1}^{\prime}(b)>l_{1}(b)$ and $F_{1}\left(l_{1}^{S}(b)\right)>F_{2}\left(l_{2}^{A}(b)\right)$. Therefore $P_{A}^{l}>P_{S 1}^{l}$ is deduced from the fact that when bidders have valuations drawn from $\left(G_{1}, G_{2}\right)$ both bidders' bid probability distributions stochastically dominate the bid probability distributions obtained when valuations are drawn from $\left(G_{1}, G_{1}\right)$. Finally, (iv) follows by (applying the arguments in (iii) to $\left(J_{1}, J_{2}\right)=\left(G_{2}, G_{2}\right)$ and. $\left(J_{1}, J_{2}^{\prime}\right)=\left(G_{2}, G_{1}\right)$.

\section{REFERENCES}

Bernhardt, D. (1995).“Toeholds and Takeovers,” mimeo, Queen's University.

Bulow, J. I., and Roberts, D. J. (1989). "The Simple Economics of Optimal Auctions," J. Polit. Econ. 97, 1060-1090.

Bulow, J. I., Huang, M., and Klemperer, P. (1999). "Toeholds and Takeovers," J. Polit. Econ. 107, 427-454.

Burkart, M. (1995). "Initial Shareholdings and Overbidding in Takeover Contests," J. Finance 5, 1491-1515.

Cramton, P., Gibbons, R., and Klemperer, P. (1987). "Dissolving a Partnership Efficiently," Econometrica 55, 615-632.

Engelbrecht-Wiggans, R. (1994). "Auctions with Price-Proportional Benefits to Bidders," Games Econom. Behavior 6, 339-346.

Lebrun, B. (1999). "First Price Auctions in the Asymmetric N Bidder Case," Int. Econ. Reu 40, 125-143.

Lebrun, B. (1998). "Comparative Statics in First Price Auctions," Games Econom. Behavior 25, 97-110.

Marshall, R., Meurer, M., Richard, J-F., and Stromquist, W. (1994). "Numerical Analysis of Asymmetric First Price Auctions," Games Econom. Behavior 7, 193-220.

Maskin, E., and Riley, J. G. (1985). "Auction Theory with Private Values," Ame. Econ. Reu. 75, 150-155.

Maskin, E., and Riley, J. G. (1999). "Asymmetric Auctions," Reu. Econ. Stud., forthcoming.

McAfee, P. (1992). "Amicable Divorce: Dissolving a Partnership with Simple Mechanisms," J. Econ. Theory 56, 266-293.

Shaked, M., and Shanthikumar, J.G. (1994). Stochastic Orders and their Applications. New York: Academic Press.

Singh, R. (1995). "Takeover Bidding with Toeholds: The Case of the Owner's Curse," mimeo, Washington University at St. Louis. 